\title{
Research on dynamic modeling of single leg of Wheeling \& walking electric vehicle based on RecurDyn
}

\author{
Junwen Xing ${ }^{a}$, Zhen Dong ${ }^{b,}$, Wenbin Chai ${ }^{c}$ and Zhenxing Li $^{d}$ \\ The Academy of Armored Forces Engineering, Beijing 100072, China

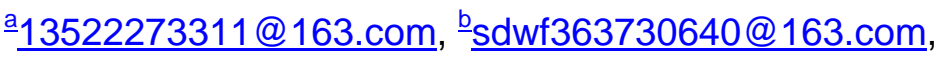 \\ 1ㅗㄷ22677244@163.com, $\underline{\text { 363730640@qq.com }}$
}

\begin{abstract}
In order to analyze the performance of the single leg of the electric vehicle in depth, a simulation method based on the digital virtual prototype software RecurDyn is proposed. The kinematics characteristics of single leg of the experimental Wheeling \& walking electric vehicles are studied systematically. The dynamic model of SolidWorks is introduced into RecurDyn to simulate and analyze the dynamics, the mathematical model created by MATLAB is used to verify the correctness of RecurDyn. The research method gives the integrated evaluation of the performance of the single leg from the system point of view, which improves the speed and precision of the product development and reduces the development cost.
\end{abstract}

Keywords: Wheeling \& walking electric vehicle, RecurDyn, Dynamic modeling, MATLAB.

\section{Introduction}

There are many problems exist in traditional physical prototype deployment pattern such as long development cycle, high cost and difficult to modify. The application of mathematical modeling method can effectively overcome the defects of the traditional physical prototype development pattern, accelerate the development speed, save the development cost, and provide an effective means for designing and evaluating the product of the whole system and performance. As one of the representative products in the UGV, the wheeling \& walking electric vehicle is one of the typical unmanned aerial vehicles with typical function, complex structure and wide application. It plays an important role in the military field. Practice shows that the gait walking is one of the most common and the most frequent working modes of the wheeling \& walking electric vehicle, which is achieved mainly by its walking mechanism. The structure and design principle of the walking mechanism is directly related to the reliability, stability and efficiency of the whole system. Therefore, the study of this part has important theoretical value and practical significance. We establish the dynamic model of a single leg though the application of virtual prototype technology to simulate the dynamic performance, which can greatly shorten the product development cycle, reduce product development costs, improve the system-level performance and quality of the product as well as get the most optimized and innovational of product. In this paper, the dynamic simulation of the single leg of the wheeling \& walking electric vehicle is studied by using the digital prototype technology RecurDyn, and the simulation results are carried out by MATLAB to verify the correctness of the results. Organization of the Text.

\section{The brief introduction of RecurDyn}

RecurDyn (Recursive Dynamic) is a new generation of multi-body system dynamics simulation software developed by the Korean company FunctionBay, it uses the relative coordinate equations and complete recursive algorithm, which is very suitable for solving large-scale problems of multibody system dynamics. The traditional dynamic analysis software is far from perfect to solve the common problem of contact collision exist in mechanism, too much simplification, low solving efficiency, poor stability which is difficult to meet the need of engineering application. So South Korea company FunctionBay make full use of the latest multi-body dynamics theory, develop the 
software RecurDyn based on the relative coordinate system modeling and recursive solution. RecurDyn can not only solve the problems of traditional kinematics and dynamics, but also solve the problem of contact collision in engineering. In view of the strong solution function of software RecurDyn, it has been widely used in aerospace, military vehicles, military equipment, engineering machinery, electrical equipment, entertainment equipment, automobile and truck, railway, ship machinery and other general machinery industry.

\section{Single leg of the wheeling \& walking electric vehicle}

In this paper, the single leg is the basic unit of the realization of the walking electric vehicle movement, each unit body includes: hip, thigh, calf, steering mechanism, wheels and 3 hydraulic actuators, which is shown in figure1.

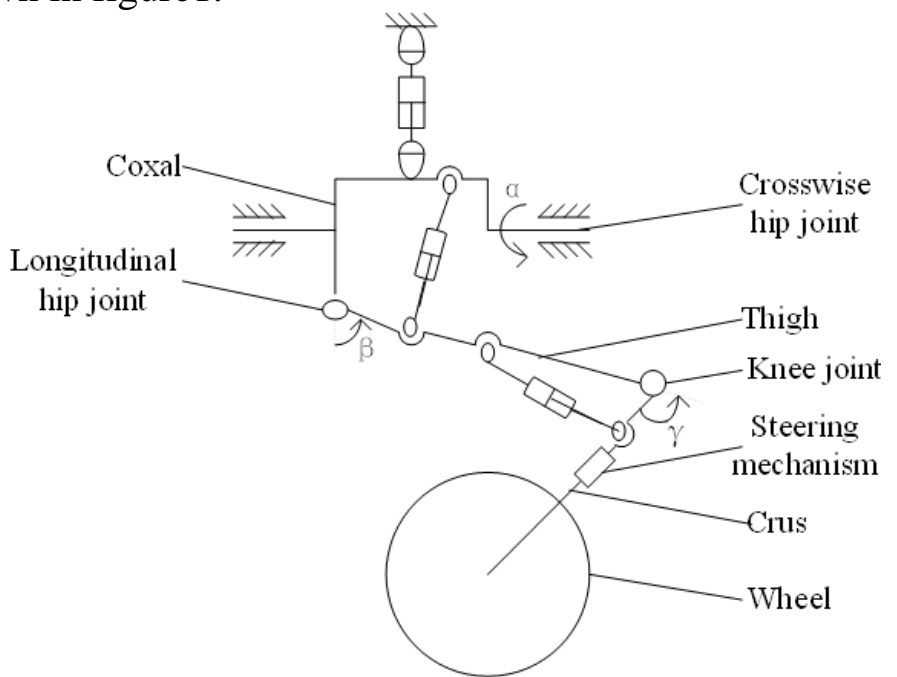

Fig. 1. The principle diagram of walking mechanism of the single leg

The hip, thigh and calf are sequentially connected by 2 transverse hinge pins to form the basic frame of the leg. The pith is attached to the frame by one hinge pins which is longitudinal with the frame, it acts as a rotating shaft in the hip and limb movement, and is the actual rotational joints of the wheeling \& walking electric vehicle. The output shaft sleeves of the 3 hydraulic actuators are respectively connected with the rotating parts of the hip, thigh and calf. Figure 2 is the required physical map for a single leg the RecurDyn needed to simulate.

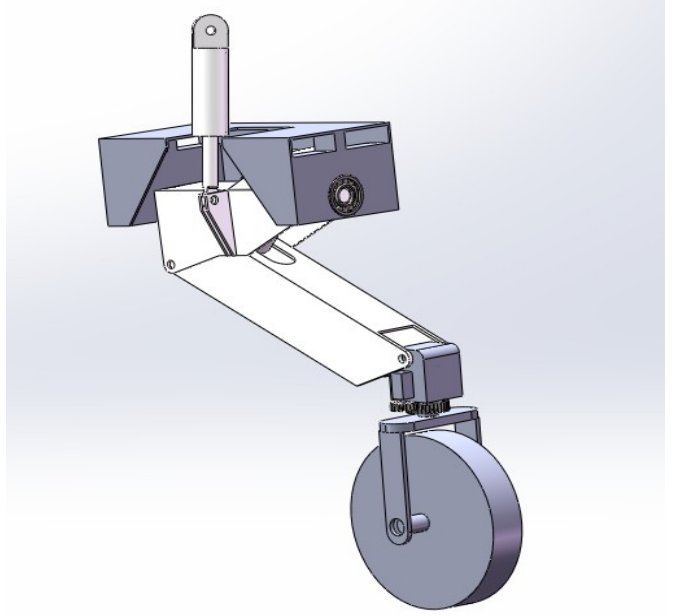

Fig. 2. The physical map for a single leg of the wheeling \& walking electric vehicle

Firstly, in this paper, we make a dynamic simulation of the single leg of the wheeling \& walking electric vehicle using RecurDyn. Then establish linkages between the desired trajectory and the corresponding target rotation sequence of each working device, give the 3 hinges certain angular velocity and angular acceleration respectively. Next, establish the dynamic model of each working 
device though Lagrange equation. Finally, the correctness of the simulation for RecurDyn is checked and verified by MATLAB.

\section{The analysis of dynamic simulation of single leg for wheeling \& walking electric vehicle}

\subsection{Module imports of RecurDyn}

RecurDyn itself has a simple geometric modeling tools for the establishment of general cylindrical, rectangular, ball, rod and conveyor belt, chain, gear, etc. But for complex geometry encountered in the practical application, we need to establish the geometry in specialized modeling software in order to facilitates positioning size, obtain high precision geometry, and improv the precision of virtual simulation for RecurDyn. In this paper, we use the SolidWorks to establish each parts of the single leg working device of the wheeling \& walking electric vehicle, and save the file as the format of Parasolid after assembly. Finally, we import the RecurDyn to add attributes, constraints and drivers for the geometric model.

\subsection{The analysis of dynamic simulation}

For a given driving force or torque for each articulated link, it is not only related to the dynamic process of the movement, but also depends on the properties of the joints (including the joint material, geometry, centroid position, inertia, etc.). The steering of the calf and the wheel drive are not considered during its walking. The single leg has 3 degrees of freedom. The kinetic study includes the establishment of dynamic model, stress analysis, inertia force calculation, dynamic balance, dynamic response. The dynamic modeling is the most important within many kinetics problems. When the joints are moving in the given condition, the force of each drive rod will change over time. Whether the force of each joints changing gently, the size of force meeting requirements makes great significance to the design and control of the mechanism. In the research of the dynamic modeling of the 3-degree of freedom for the single leg, the torque of the 3 angulations is used as the simulation object, the independent movement of each rotating hinge is imposed, and the rotational torque is simulated by the joint rotation of the rotation angle of $1-3$.

Firstly, we need to add 3 rotating hinge joint to the geometric model of the 3-degree of freedom single leg. Then open the properties of the 3 "Revolute" on the right side of the "Database", modify the name and add "Motion", each "Motion" establish the expression according to the movement mode. Next, click on the upper side of the operating interface "Analysis" to run the simulation program. Finally, click on "Plot Result" to derive a series of rotating drive torque curve. The drive torque curve of the 3 corners is shown in figure 3.

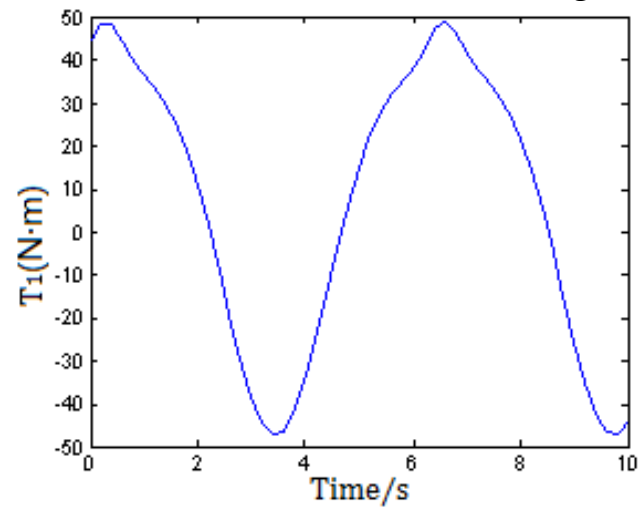

(a)

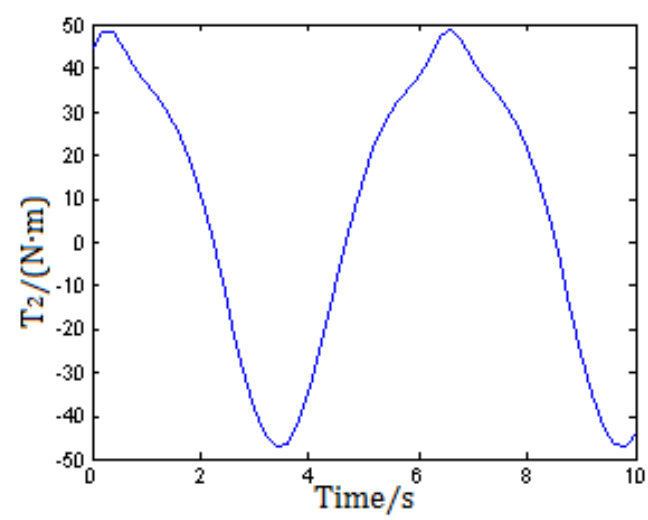

(b) 


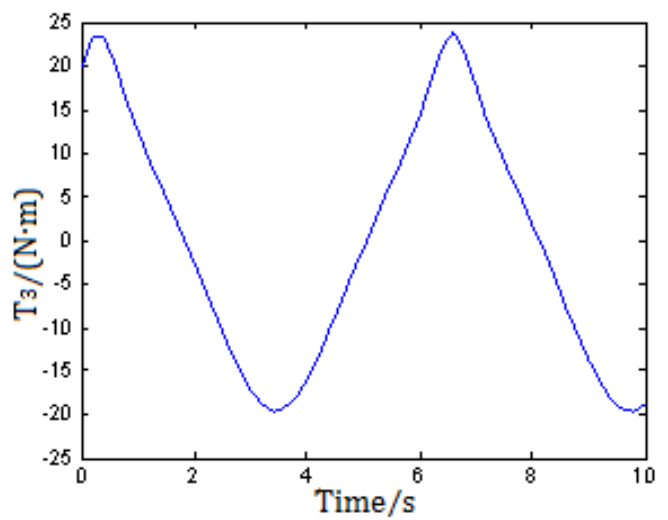

(c)

Fig. 3. The torque diagram with 3 rotation angles as variables at the same time

\subsection{The application of MATLAB to check}

In order to check the correctness of the 3-degree of freedom dynamics simulation of the Single leg in RecurDyn, the Matlab software is used. In order to facilitate the analysis of the single leg of the wheeling \& walking electric vehicle, It is needed to establish a relationship with the coordinate system. The coordinate system of the 3-degree of freedom mechanism can be established according to the $\mathrm{D}-\mathrm{H}$ method in the space mechanism, The coordinate system of the single leg includes the base coordinate system, the hip joint coordinate system, the thigh joint coordinate system and the calf joint coordinate system, which are respectively represented as $\{0\},\{1\},\{2\}$ and $\{3\}$, as shown in figure 4 .

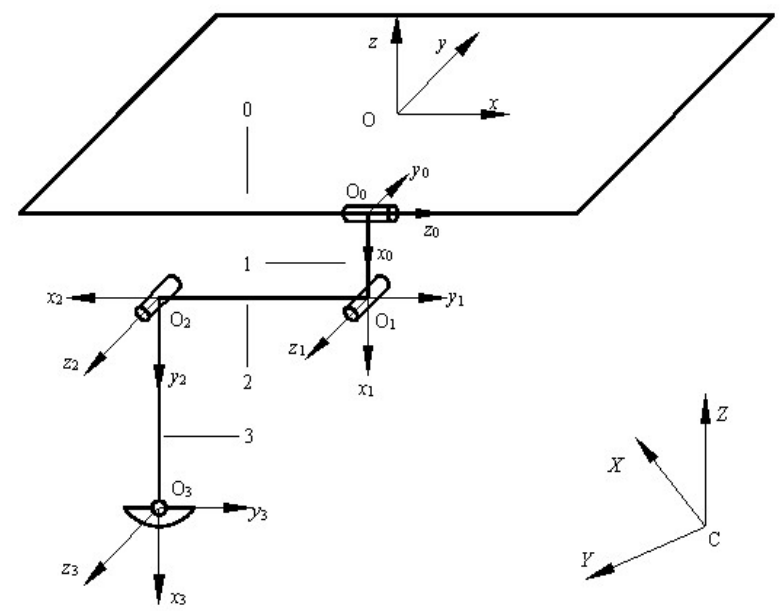

Fig. 4. The D-H coordinate system of the single leg of the wheeling \& walking electric vehicle

Firstly, establish linkages between the desired trajectory and the corresponding target rotation sequence of each working device, give the 3 hinges certain angular velocity and angular acceleration respectively. Then, the dynamic model of each working device is established by Lagrange equations, and a series of torque curves are derived by the calculation of MATLAB. Finally, compare the calculation and simulation results. The calculated results of MATLAB for the 3 rotational torque are shown in Figure 5.

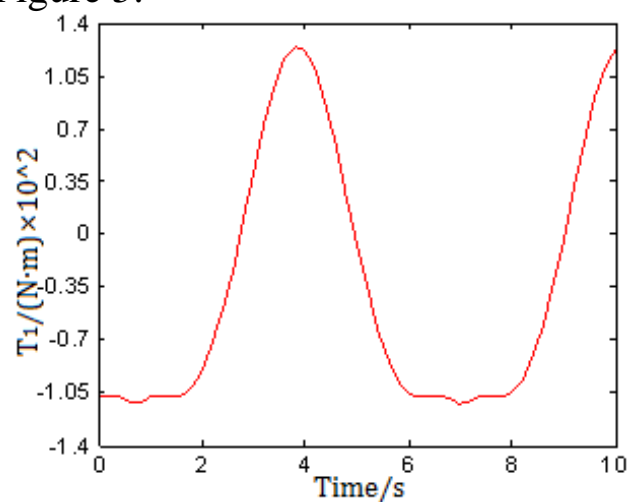

(a)

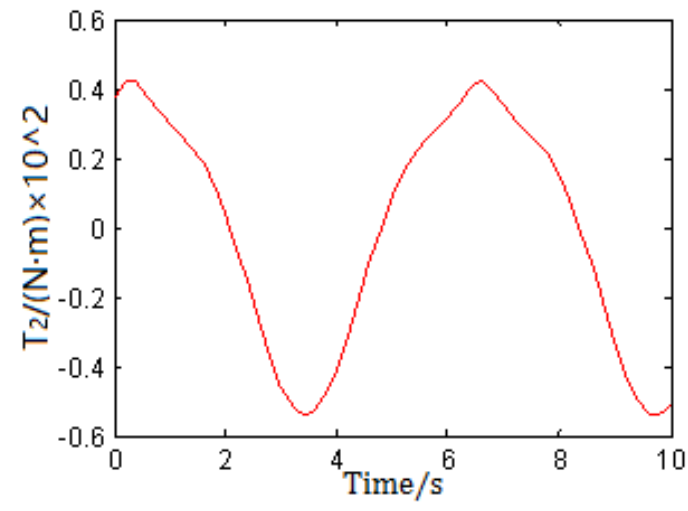

(b) 


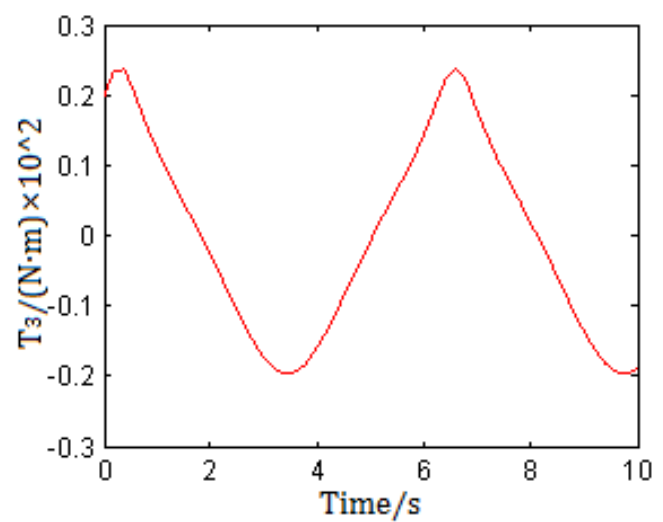

(c)

Fig. 5. The torque diagram of the 3 corners based on MATLAB

It is obvious that the simulation results of the 2 methods are the same by comparing figure 3 and figure 5. However, the operation of modeling and simulation of RecurDyn is not only simple, but also can get many curves of kinematics and dynamics while MATLAB requires a lot of computation, which is troublesome, and the first calculation can't get all the kinematics and dynamics results.

\section{Conclusion}

The application of modeling the single leg of the wheeling \& walking electric vehicle using RecurDyn can combine the single leg modeling, kinematics analysis, dynamics analysis and other aspects organically. Realizing The dynamic analysis of the single leg mechanism, speeding up the product development, Saving the development cost, providing an effective means for the design and evaluation of the product for the whole system.

\section{References}

[1] Xiaojuan Jiao, Jiewei Zhang, Binbin Peng. Multi body system optimization simulation technology of RecurDyn. M. Beijing: Tsinghua University press, (2010)

[2] Li Ma. A practical Course on MATLAB language. M. Beijing: Tsinghua University press, (2010)

[3] Jingwei Hou. Study on the dynamic modeling of 4-degree of freedom hydraulic robot based on RecurDyn. J. Hoisting and conveying machinery, 3(2011): 51 53.

[4] Xuedong Chen, Hongxun Guo, Watanabe Keigo. The forward kinematics analysis of quadruped robot crawling. J. Journal of mechanical engineering, 39(2003): 8 12.

[5] Hongbo Wang, Zhen Huang. Lagrange dynamic method for six degree of freedom parallel robot. J. Robot, 1(1990): 23 26.

[6] Feng Yu. Research on the dynamics of special robot based on the virtual prototype technology. D. Master Thesis of Lanzhou University of Technology, (2003):6. 